\title{
DNA Markers Linked to Eastern Filbert Blight Resistance from a Hazelnut Selection from the Republic of Georgia
}

\author{
Vidyasagar R. Sathuvalli, Shawn A. Mehlenbacher ${ }^{1}$, and David C. Smith \\ Department of Horticulture, Oregon State University, 4017 Ag \& Life Sciences Building, Corvallis, \\ OR 97331
}

\begin{abstract}
AdDITIONAl INDEX wORDS. disease resistance, Anisogramma anomala, marker-assisted selection, simple sequence repeats, filbert

Abstract. The hundred-year history of the european hazelnut (Corylus avellana L.) industry in the Pacific northwestern United States is threatened by eastern filbert blight (EFB) caused by the fungus Anisogramma anomala (Peck) E. Müller. Marker-assisted selection has been extensively used for 'Gasaway' resistance in the hazelnut breeding program at Oregon State University. Concern over possible breakdown of this single resistance gene provides an incentive to look for new sources of resistance. OSU 759.010, a selection from the Republic of Georgia, has remained free of EFB after inoculations over several years. Random amplified polymorphic DNA (RAPD) markers linked to resistance were identified by screening primers against three resistant seedlings, three susceptible seedlings, and the parents of a segregating seedling population. For the progeny OSU 759.010 $\times$ OSU 653.068, 13 linked markers were identified. The markers most closely linked to resistance were 695-1800 on the proximal side and H12-640, 373700, 349-450, and F08-700 on the distal side. Four of the five markers also segregated in the progeny OSU $759.010 \times$ OSU 665.076, whereas H12-640 was monomorphic. Segregation for disease response in the first population showed a surplus of resistant seedlings, approaching a 3:1 ratio, with closely linked RAPD markers showing similar ratios. In the second population, the observed segregation for disease response and associated markers did not deviate from the expected 1:1 ratio. Based on cosegregation with simple sequence repeat (SSR) markers, resistance from OSU 759.010 was assigned to linkage group 2. Resistance to EFB from 'Gasaway' and 'Ratoli' was previously mapped to linkage groups 6 and 7, respectively. Therefore, OSU 759.010 provides a novel source of EFB resistance and markers 6951800, 373-700, 349-450, and F08-700 have potential for use in marker-assisted selection to pyramid EFB resistance alleles.
\end{abstract}

Hazelnut, also known as filbert, is the fruit of plants in the genus Corylus L., a member of the family Betulaceae. The european hazelnut is a deciduous shrub or small tree native to most of Europe and adjacent areas in the Caucasus Mountains and Asia Minor. Major producers of hazelnuts are Turkey, Italy, the United States, Spain, Azerbaijan, Georgia, and France. With commercial production centered in Oregon's Willamette Valley (Hazelnut Marketing Board, 2004), the 37,100 t produced in the third-ranked United States represents $3.4 \%$ of the world's hazelnuts (Food and Agriculture Organization of the United Nations, 2009).

One threat to Oregon's hazelnut industry is the fungal disease EFB caused by the pyrenomycete Anisogramma anomala. The fungus is an obligate biotroph with a 2-year life cycle (Pinkerton et al., 1995) that infects several species in the genus Corylus. On the commercially important european hazelnut, the fungus causes severe stem cankers leading to rapid yield losses and to eventual tree death in 5 to 12 years if proper control measures are not taken (Pinkerton et al., 1993). The pathogen is endemic to eastern North America where the wild american hazel (Corylus americana Marsh.) is its host. The life cycle of the pathogen is well documented (Johnson et al., 1996; Pinkerton et al., 1992, 1998a, 1998b, 2001; Stone et al., 1992). Ascospores released from perithecia are dispersed by splashing rains with active discharge occurring during prolonged

Received for publication 2 June 2011. Accepted for publication 7 July 2011. ${ }^{1}$ Corresponding author. E-mail: mehlenbs@hort.oregonstate.edu. periods of branch wetness. The ascospores germinate and produce hyphae that directly penetrate young growing shoots, permeate and destroy the cambial layer, and eventually produce visible cankers having ascospores within stromata 12 to 16 months after infection. EFB in the Pacific Northwestern United States was first diagnosed in 1970 after being noticed in 1968 by a grower in southwestern Washington (Davison and Davidson, 1973). Since its discovery, EFB has moved southward at an average rate of 2 to $3 \mathrm{~km}$ per year (Pinkerton et al., 1995). It is now present throughout the Willamette Valley. Current control measures include scouting and pruning of the infected branches $30 \mathrm{~cm}$ below the cankers and routine fungicide treatments beginning at budbreak and continuing at 2-week intervals during growth of new shoots (Pscheidt, 2006). Because of environmental concerns over the use of fungicides and the high cost incurred in applications, host genetic resistance is viewed as the most desirable and economical means of controlling this disease (Mehlenbacher, 1994).

Complete resistance to EFB was first discovered in 'Gasaway', an obsolete pollenizer that was found free of symptoms in a heavily infected 'DuChilly' orchard (Cameron, 1976). Resistance from 'Gasaway', conferred by a dominant allele at a single locus (Mehlenbacher et al., 1991), has been extensively used in the hazelnut breeding program at Oregon State University (OSU). Most resistant selections from the breeding program carry 'Gasaway' resistance. In greenhouse inoculation studies with various isolates of $A$. anomala, Molnar et al. (2010a, 2010b) demonstrated that an isolate from Michigan was 
able to infect 'Gasaway', emphasizing the importance of using more than one source of resistance in breeding. Greenhouse inoculation studies (Chen et al., 2007; Coyne et al., 1998; Lunde et al., 2000; Sathuvalli et al., 2010) identified several $C$. avellana selections resistant to EFB. Scions received from the Republic of Georgia under the names 'Tskhenis dzudzu' and 'Gulshishvela' showed no signs or symptoms of the fungus after a greenhouse inoculation in 1998 (S.A. Mehlenbacher, unpublished data). The trees had identical phenotypes and are maintained under the designation OSU 759.010. The selection has remained free of infection in a series of greenhouse inoculations from 1998 to 2004. Nuts of OSU 759.010 are early-maturing, small to medium in size, round with a slight point, and some of the nuts show suture splits. The trees bear heavy crops of nuts in alternate years, although the kernels fill the nuts poorly when crop load is heavy. The catkins drop to the ground in early fall, making the selection functionally malesterile. Lasareishvili (2003) described 'Tskhenis dzudzu' as a minor Georgian cultivar with large, long nuts, whereas 'Gulshishvela', which is widely grown in the Guria region, has bushes that are vigorous, precocious, and highly productive with regular annual crops. 'Gulshishvela' nuts are small and nearly round, and the shells are dark brown. When the two pairs of trees in our collection labeled 'Tskhenis dzudzu' and 'Gulshishvela' began to bear nuts, both trees in each pair appeared identical, but neither pair was true-to-name. The identity of OSU 759.010 remains unknown, although it most closely resembles 'Gulshishvela'. True-to-name 'Gulshishvela', received at a later date, is susceptible to EFB (Sathuvalli et al., 2010). OSU 759.010 and 'Gulshishvela' have incompatibility alleles $\mathrm{S}_{4}$ and $\mathrm{S}_{20}$ (S.A. Mehlenbacher, unpublished data), which supports a common ancestry.

Current EFB evaluation methods are slow and time-consuming, requiring 16 to 20 months to identify resistant cultivars and seedlings. Molnar et al. (2005) proposed an accelerated screening method that merits evaluation in the field on a large scale. Identification of molecular markers closely linked to EFB resistance alleles would greatly facilitate the development of new cultivars through marker-assisted selection (MAS). RAPD markers produced by the polymerase chain reaction (PCR) are one of the least expensive types of DNA markers and are suitable to the high sample throughput required for routine use in applied breeding programs (Welsh and McClelland, 1990; Williams et al., 1990). RAPD markers are dominant, easy to assay, require only a modest investment in laboratory equipment, and are amenable to automation. However, the RAPD technique is sensitive to amplification conditions such as concentrations of primers, $\mathrm{MgCl}_{2}$, DNA template, and Taq polymerase as well as denaturation temperature (Devos and Gale, 1992). Furthermore, Meunier and Grimont (1993) showed variations in RAPD banding patterns associated with the brand of Taq polymerase and the model of thermal cycler. While recognizing these limitations, robust RAPD markers have been useful for many applications, including MAS. In hazelnut, Mehlenbacher et al. (2004) identified 20 RAPD markers linked in coupling and five markers linked in repulsion to 'Gasaway' resistance. Sathuvalli et al. (2011) identified four RAPD markers, three in coupling and one in repulsion, linked to 'Ratoli' resistance. Markers 152-800 and 268-580, which flank the 'Gasaway' resistance allele, and marker G17-800, which is closely linked to a second resistance allele derived from cultivar Ratoli, are being used in MAS. Only seedlings having at least one marker are planted in the field. The markers linked to resistance in 'Gasaway' and 'Ratoli' are absent in OSU 759.010.

The assignment of resistance loci to different linkage groups (LGs) confirms their independence and thus facilitates their use in pyramiding. To date, more than 230 SSR marker loci have been developed in hazelnut (Bassil et al., 2005a, 2005b; Boccacci et al., 2005; Gürcan and Mehlenbacher, 2010; Gürcan et al., 2010), and most have been assigned to linkage groups using the mapping population of Mehlenbacher et al. (2006). These SSRs are useful for assigning additional loci to LGs. Mehlenbacher et al. (2006) assigned EFB resistance from 'Gasaway' to LG6, whereas Sathuvalli et al. (2011) showed that resistance from the Spanish cultivar Ratoli is on LG7 based on cosegregation with mapped SSR markers.

In this study, we examined segregation for disease response in two progenies from crosses of OSU 759.010 with susceptible selections, identified linked RAPD markers, and assigned the resistance locus to a LG based on cosegregation with mapped SSR markers.

\section{Materials and Methods}

Plant materials. In 2001, two controlled crosses were made between OSU 759.010 as the female parent and EFBsusceptible selections OSU 653.068 and OSU 665.076 as the male parents, generating 154 and 149 seedlings in progenies designated 01032 and 01033 (Fig. 1), respectively. From these populations, subsets were used for disease inoculations: 89 seedlings from progeny 01032 and 69 seedlings from progeny 01033. For marker segregation and mapping, 94 seedlings from progeny 01032 and 85 seedlings from progeny 01033 were used. The seedlings and parents were grown in the field at the OSU Smith Horticulture Research Farm in Corvallis, OR.

Greenhouse inoculation. Disease inoculations were carried out in a greenhouse and later the inoculated trees were planted in the field at the Smith Horticulture Research Farm. Cankered shoots with mature stromata were collected at the North Willamette Research and Extension Center in Aurora, OR, in Dec. 2004. They were stored at $-20{ }^{\circ} \mathrm{C}$ until used as a source of inoculum. Scion wood was collected from seedlings and their parents in Jan. 2005 and stored at $-1{ }^{\circ} \mathrm{C}$ until grafting. Three trees of each seedling were grafted in Apr. 2005. The grafted plants were grown in 5-L pots containing a mixture of equal volumes of peat, pumice, fine bark dust, and $9 \mathrm{~g}$ of 3- to 4-month-release 18N-2.6P-10K fertilizer (Sierra; Peters Professional, Allentown, PA). The grafted trees were grown in a greenhouse under optimal conditions [24/18 ${ }^{\circ} \mathrm{C}$ (day/night)] until ready for inoculation.

Inoculation chambers were set up in the greenhouse using polyvinyl chloride tubing (1.27 cm diameter) placed on top of benches $(2.44 \times 0.88 \mathrm{~m})$ and covered with white $0.1-\mathrm{mm}$ polythene sheeting with the roof opened. High humidity was maintained using misters. Three misters $\left(7.6 \mathrm{~L} \cdot \mathrm{h}^{-1}\right)$ per bench were placed $0.3 \mathrm{~m}$ apart at a height of $0.9 \mathrm{~m}$ above the bench top and set to operate for $10 \mathrm{~s}$ every $30 \mathrm{~min}$ during the daytime (0800 to $1900 \mathrm{HR}$ ) and $10 \mathrm{~s}$ every hour during the night (1900 to $0800 \mathrm{HR}$ ) using an automated misting modifier (Model No. DE 8 PR2; Davis Engineering, Canoga Park, CA). Grafted plants were inoculated when the shoots had four to five nodes (Coyne et al., 1996) and actively growing shoot tips. Perithecia from the diseased twigs were dissected and ground with a mortar and pestle to release ascospores. The spores were diluted with 

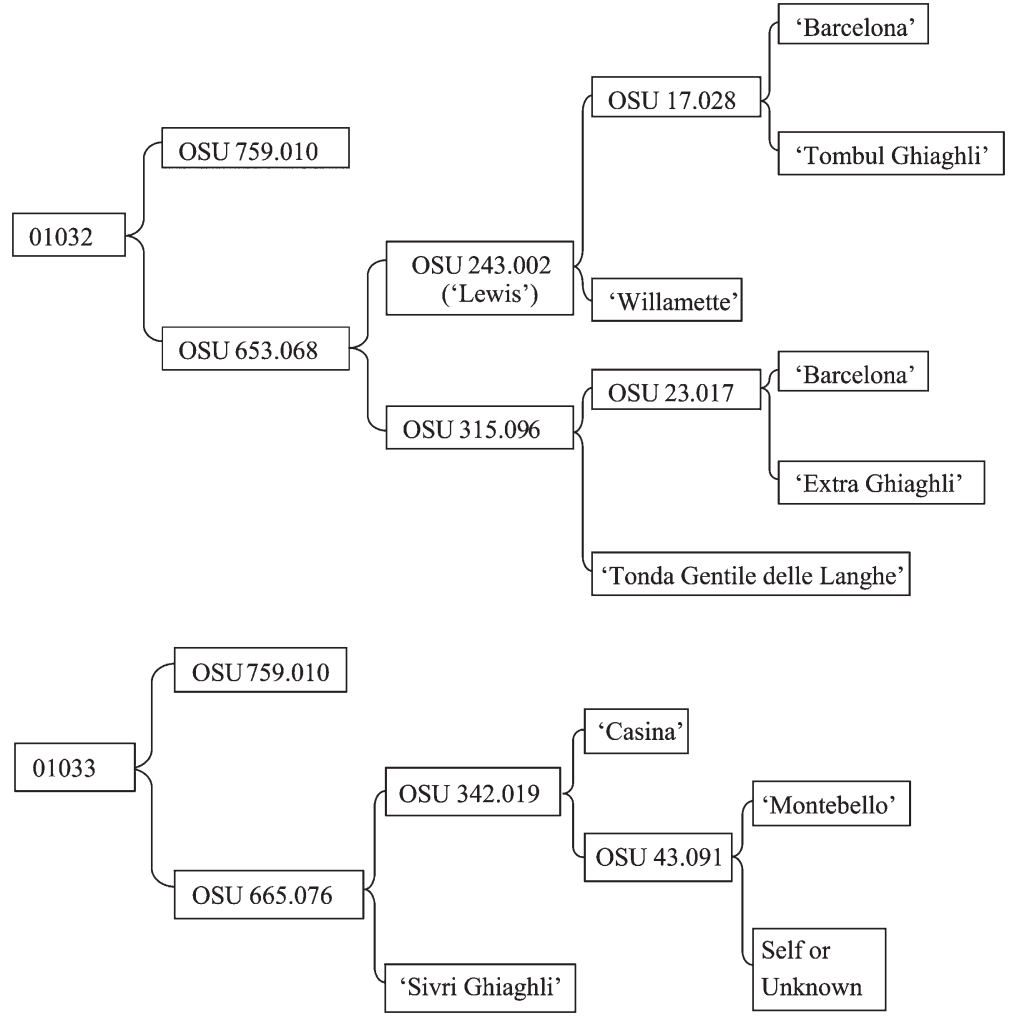

Fig. 1. Pedigrees of Corylus avellana progenies 01032 and 01033 segregating for resistance to eastern filbert blight from Georgian selection OSU 759.010. All selections other than OSU 759.010 are susceptible.

distilled water to a concentration of $1 \times 10^{6}$ spores $/ \mathrm{mL}$ for use in inoculation. Two inoculations at a $3-d$ interval were carried out either in the evening ( 2000 or $2200 \mathrm{HR}$ ) or morning (0500 or $0700 \mathrm{HR}$ ) to reduce the risk of escapes. The spore suspension was sprayed until the shoot tips were almost at the point of runoff. The inoculated trees were moved out of the inoculation chamber $3 \mathrm{~d}$ after the second inoculation and grown in the greenhouse at optimal temperatures $\left[24 / 18{ }^{\circ} \mathrm{C}\right.$ (day/night)] for 3 months and then planted in a nursery row in the field. The susceptible cultivar Ennis, susceptible parents OSU 653.068 and OSU 665.076, and resistant parent OSU 759.010 were included as controls.

Disease Susceptibility EVAluation. The inoculated plants were evaluated in the field for the presence of cankers 16 to 20 months after inoculation. A genotype was scored as susceptible if cankers with stromata were noticed in one or more of the three trees and scored as resistant if all three trees remained free of infection for more than 20 months.

DNA EXTRACTION. Two methods of DNA extraction were used. For initial screening of primers, large quantities of DNA template were essential. From progeny 01032 , fresh young leaves from five susceptible seedlings, five resistant seedlings, and the parents were collected during Spring 2005. Leaves were ground under liquid nitrogen and kept at $-80{ }^{\circ} \mathrm{C}$ until extraction. DNA from ground leaves was extracted using a Puregene DNA isolation kit (Gentra Systems, Minneapolis, MN) according to the manufacturer's instructions. For mapping, fresh young leaves of 96 seedlings of progeny 01032 and 85 seedlings of progeny 01033 were collected from the field in Spring 2005. DNA was extracted from these leaves following the method of
Lunde et al. (2000) with no RNAase treatment. The DNA extracted by both methods was quantified using a spectrophotometer (Nano Drop TM ND-1000; NanoDrop Technologies, Wilmington, DE) and diluted with TE buffer to a concentration of 5 to 25 ng. $\mu \mathrm{L}^{-1}$.

RANDOM AMPLIFIED POLYMORPHIC DNA ANALYSIS. Three resistant seedlings and three susceptible seedlings of progeny 01032 and the parents were used for primer screening. A total of 900 primers was screened: all primers in kits $\mathrm{AF}$ to $\mathrm{AZ}$ and selected primers in sets $A$ to AE from Eurofins MWG Operon (Huntsville, AL) and 380 selected primers in sets 1 to 800 from the Michael Smith Laboratories of the University of British Columbia (UBC, Vancouver, BC, Canada). The selected primers from Operon Technologies and UBC were those that generated polymorphic markers in a population segregating for resistance from 'Gasaway' (Mehlenbacher et al., 2006). Primers that generated a band present in the resistant parent and all three resistant seedlings but absent in the susceptible parent and all three susceptible seedlings were further investigated as markers linked to resistance in coupling. Primers that generated a band present in the resistant parent and all three susceptible seedlings but absent in the susceptible parent and all three resistant seedlings were further investigated as markers linked to resistance in repulsion. Markers that showed recombination in one of six seedlings in the initial screening were further investigated in a group of 24 additional seedlings of the progeny 01032. Markers that showed less than 30\% recombination with resistance in the 24 seedlings were amplified in the remaining seedlings of the population.

PCRs were performed in a $15-\mu \mathrm{L}$ volume containing $0.4 \mu \mathrm{M}$ of primer, 5 to $25 \mathrm{ng}$ of template DNA, $0.4 \mathrm{U}$ of Biolase DNA polymerase (Biolase USA, Randolph, MA), $1.5 \mathrm{~mm} \mathrm{MgCl}_{2}$, $120 \mu \mathrm{M}$ each of dATP, dCTP, dGTP, and dTTP, and the $1 \times$ ammonium-based buffer supplied by the manufacturer (Mehlenbacher et al., 2004). Ninety-six reactions were run simultaneously using Geneamp ${ }^{\circledR}$ PCR System 9700 thermal cyclers (Perkin-Elmer Corp., Foster City, CA). The thermal cycler program consisted of an initial $5 \mathrm{~s}$ at $95^{\circ} \mathrm{C}$ and $1 \mathrm{~min} 55 \mathrm{~s}$ at $92{ }^{\circ} \mathrm{C}$ followed by 40 cycles of $5 \mathrm{~s}$ at $95{ }^{\circ} \mathrm{C}, 55 \mathrm{~s}$ at $92{ }^{\circ} \mathrm{C}$, $1 \mathrm{~min}$ at $35^{\circ} \mathrm{C}, 2 \mathrm{~min}$ at $72{ }^{\circ} \mathrm{C}$, and then $7 \mathrm{~min}$ at $72{ }^{\circ} \mathrm{C}$ ending with an indefinite hold at $4{ }^{\circ} \mathrm{C}$ until retrieved from the thermal cycler. During the first five cycles, the ramp time from 35 to $72{ }^{\circ} \mathrm{C}$ was reduced to $30 \%$ of maximum to minimize nonspecific binding of primers (Mehlenbacher et al., 2004). When necessary to improve repeatability of scoring, primer and $\mathrm{MgCl}_{2}$ concentrations were adjusted. Amplification products were separated by electrophoresis on $2 \% \mathrm{w} / \mathrm{v}$ agarose (ISC Bioexpress, Kaysville, UT), stained with ethidium bromide (Sigma-Aldrich, St. Louis, MO), and photographed using an ultraviolet imaging system (UVP, Upland, CA).

Simple SEQuence REPEAT ANALYSIS. A total of 102 SSR markers were chosen from those previously identified (Bassil et al., 2005a, 2005b; Boccacci et al., 2005; Gürcan and 
Mehlenbacher, 2010; Gürcan et al., 2010) based on polymorphic information content, ease of scoring, and suitability for multiplexing. SSR primer pairs were initially screened against the parents of progeny 01032 (OSU 759.010 and OSU 653.068) to identify polymorphic markers. The 34 SSR markers that were polymorphic between the parents, and heterozygous in OSU 759.010, were used for cosegregation analysis with disease phenotype. Each allele at each SSR locus was scored as present (1) or absent (0). Disease response was scored similarly, resistant (1) or susceptible (0). Initially, 30 seedlings (15 resistant and 15 susceptible) were genotyped with SSRs as described by Gürcan and Mehlenbacher (2010) and Gürcan et al. (2010). Correlation coefficients were calculated using the PROC CORR of SAS (Version 9.2; SAS Institute, Cary, NC). A correlation coefficient of disease phenotype and SSR marker score greater than 0.5 indicated linkage to the disease resistance locus. Because the SSR markers had been previously mapped (Gürcan and Mehlenbacher, 2010; Gürcan et al., 2010; Mehlenbacher et al., 2006), once the LG carrying the resistance locus had been identified, all SSR markers in the LG were used for mapping.

DAta AnAlysis. Segregation analysis for EFB resistance in the two progenies was performed using a chi-square goodnessof-fit test. A test of heterogeneity was also performed to decide whether the data from the two progenies could be pooled. Markers potentially linked to disease resistance were scored on seedlings in progenies 01032 and 01033 and linkage maps constructed using JoinMap Version 4.0 (Van Ooijen and Voorrips, 2006) as described previously (Sathuvalli et al., 2011). JoinMap also was used to calculate the mean chi-square contributions of all markers in the linkage group for progeny 01032, thus allowing comparison of segregation ratios at adjacent loci.

\section{Results}

SEgREgation FOR EASTERN FILBERT BLIGHT RESISTANCE. The parent OSU 759.010 showed consistent resistance in a series of greenhouse inoculations. 'Ennis' and the susceptible parents, OSU 653.068 and OSU 665.076, showed disease symptoms after greenhouse inoculations as expected. Disease inoculations were carried out on a total of 158 seedlings. Progeny 01032 showed a ratio of three resistant:one susceptible, whereas progeny 01033 showed the expected 1:1 ratio (Table 1). The two progenies were heterogeneous, so the data were not pooled.

IDENTIFICATION OF RANDOM AMPLIFIED POLYMORPHIC DNA MARKERS LINKED TO EASTERN FILBERT BLIGHT RESISTANCE. The initial screening of 900 primers using three resistant and three susceptible seedlings from progeny 01032 identified a total of 30 markers potentially linked to EFB resistance. Of these, linkage was confirmed for 13 using 94 seedlings of progeny 01032, and a linkage map was constructed. The map spanned a distance of $72.6 \mathrm{cM}$ with 12 markers linked in coupling and one marker in repulsion (Fig. 2A). The markers are designated by the primer name followed by the size of the amplified polymorphic band. For the marker linked in repulsion, AG011700 , an $\mathrm{R}$ was placed after the band size. The resistance locus

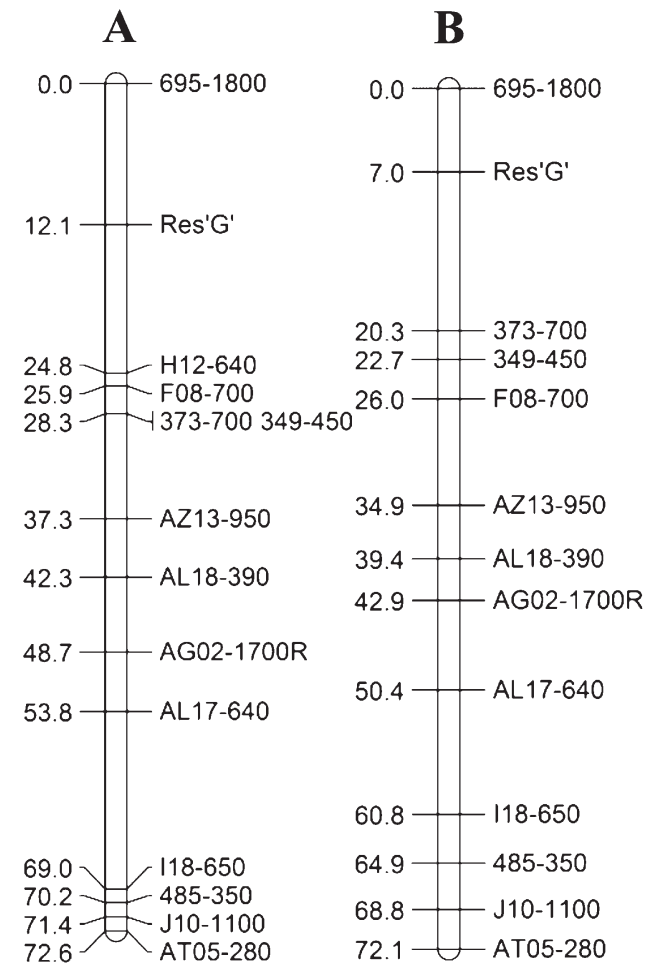

Fig. 2. Maps of random amplified polymorphic DNA markers flanking the eastern filbert blight resistance locus in Corylus avellana progenies (A) 01032 and (B) 01033 showing slight differences in distances. Markers are designated by the primer name [UBC (University of British Columbia, Vancouver, BC, Canada) or Operon (Eurofins MWG Operon, Huntsville, AL)] followed by the size of the amplified fragment. Marker AG02-1700 is linked to the others in repulsion. Marker H12-640 is monomorphic in progeny 01033 and so could not be mapped.

is flanked by markers 695-1800 and H12-640 at distances of 12.1 and $12.7 \mathrm{cM}$, respectively. Chi-square goodness-of-fit tests were performed for each marker in the linkage group for both progenies (Table 2). The four proximal markers nearest the disease resistance locus (H12-640, F08-700, 373-650, and 349450) showed a significant surplus of seedlings with the markers present but did not fit either a 3:1 or 1:1 segregation model. The single distal marker, 695-1800, and eight distant proximal markers fit a 1:1 segregation model.

To confirm the segregation and linkage of the RAPD markers, DNA from 85 seedlings of progeny 01033 were amplified with the same primers as in progeny 01032 . Except for H12-640, all primers produced polymorphic bands. H12-640 was monomorphic with a 640-bp band amplified in all seedlings. Because the disease phenotype segregated one resistant:one susceptible in 
Table 2. Chi-square goodness of fit for random amplified polymorphic DNA markers associated with eastern filbert blight resistance from Corylus avellana OSU 759.010 in progenies 01032 and $01033 .^{\mathrm{z}}$

\begin{tabular}{|c|c|c|c|c|c|c|c|}
\hline Marker $^{y}$ & Absent (no.) & Present (no.) & $\chi^{2}(1: 1)$ & $P(1: 1)$ & $\chi^{2}(3: 1)$ & $P(3: 1)$ & Best fit \\
\hline \multicolumn{8}{|c|}{ Progeny $01032=$ OSU $759.010 \times$ OSU 653.068} \\
\hline $695-1800$ & 41 & 53 & 1.53 & 0.22 & 17.38 & 0.00 & $1: 1$ \\
\hline H12-640 & 35 & 59 & 6.13 & 0.01 & 7.50 & 0.01 & - \\
\hline $349-450$ & 34 & 60 & 7.19 & 0.01 & 6.26 & 0.01 & - \\
\hline $373-650$ & 34 & 60 & 7.19 & 0.01 & 6.26 & 0.01 & - \\
\hline AZ13-950 & 40 & 54 & 2.09 & 0.15 & 15.45 & 0.00 & $1: 1$ \\
\hline AG02-1700R & 45 & 49 & 0.17 & 0.68 & 26.23 & 0.00 & $1: 1$ \\
\hline AL17-640 & 48 & 44 & 0.17 & 0.68 & 36.23 & 0.00 & $1: 1$ \\
\hline $\mathrm{I} 18-650$ & 47 & 47 & 0.00 & 1.00 & 31.33 & 0.00 & $1: 1$ \\
\hline $485-350$ & 48 & 46 & 0.04 & 0.84 & 34.06 & 0.00 & $1: 1$ \\
\hline $\mathrm{J} 10-1100$ & 47 & 47 & 0.00 & 1.00 & 31.33 & 0.00 & $1: 1$ \\
\hline AT05-280 & 48 & 46 & 0.04 & 0.84 & 34.06 & 0.00 & $1: 1$ \\
\hline $373-650$ & 26 & 59 & 12.81 & 0.00 & 1.42 & 0.23 & $3: 1$ \\
\hline $349-450$ & 28 & 57 & 9.89 & 0.00 & 2.86 & 0.09 & $3: 1$ \\
\hline AZ13-950 & 41 & 43 & 0.05 & 0.82 & 25.40 & 0.00 & $1: 1$ \\
\hline AL18-390 & 36 & 49 & 1.99 & 0.16 & 13.65 & 0.00 & $1: 1$ \\
\hline AG02-1700R & 34 & 49 & 2.71 & 0.10 & 11.28 & 0.00 & $1: 1$ \\
\hline AL17-640 & 39 & 46 & 0.58 & 0.45 & 19.77 & 0.00 & $1: 1$ \\
\hline I18-650 & 45 & 40 & 0.29 & 0.74 & 35.39 & 0.00 & $1: 1$ \\
\hline $485-350$ & 44 & 41 & 0.11 & 0.74 & 32.47 & 0.00 & $1: 1$ \\
\hline $\mathrm{J} 10-1100$ & 47 & 38 & 0.95 & 0.33 & 41.60 & 0.00 & $1: 1$ \\
\hline AT05-280 & 49 & 36 & 1.99 & 0.16 & 48.32 & 0.00 & $1: 1$ \\
\hline
\end{tabular}

${ }^{\mathrm{z} M a r k e r s ~ a r e ~ l i s t e d ~ b y ~ m a p ~ o r d e r . ~}$

y Designations for random amplified polymorphic DNA markers are the primer followed by the length of the polymorphic fragment in base pairs. Primers with three digits are from University of British Columbia, Vancouver, BC, Canada. Primers with one or two letters are from Eurofins MWG Operon, Huntsville, AL.

progeny 01033 , we expected all markers to segregate in a ratio of one present:one absent. Eight markers showed good fit to a $1: 1$ ratio. However, three markers near the resistance locus (F08-700, 373-700, and 349-450) produced bands in an excess of seedlings (Table 2). A linkage map was constructed using 12 RAPD markers and disease phenotypes (Fig. 2B). The map spanned a distance of $72.1 \mathrm{cM}$ with the resistance locus flanked by markers $695-1800$ and 373-700 at distances of 7.0 and $13.3 \mathrm{cM}$, respectively.

Correlation with segregation at previously mapped SSR markers allowed assignment to a LG of the disease response and the newly identified RAPD markers. Of the 102 initially selected SSR markers, 34 were polymorphic between OSU 759.010 and the susceptible parent OSU 653.028 and segregated in the seedling populations. Initial analysis with 15 resistant and 15 susceptible seedlings of progeny 01032 identified two SSR markers (KG811-257 and B789-176) with correlation coefficients greater than 0.5 . These two markers had been previously assigned to LG2 (Gürcan and Mehlenbacher, 2010; Gürcan et al., 2010). Segregation at five additional SSR markers (B011, B501, B789, KG811, and KG850) on LG2 was also studied in progeny 01032 and the loci placed on the map. The combined map (Fig. 3) for progeny 01032 includes five SSR markers, 13 RAPD markers, and the disease resistance locus. On the map, the closest SSR marker (B501-124) is $22.3 \mathrm{cM}$ from the resistance locus, and the furthest SSR marker (KG850-365) is placed at a distance of $103.5 \mathrm{cM}$. The mean chi-square contributions of all markers in LG2 for progeny 01032 are less than 3.0 (Table 3), confirming similar segregation ratios at nearby loci.

\section{Discussion}

With the advent of recombinant DNA technology and the PCR, different types of DNA markers became available to breeders, geneticists, and germplasm specialists (Mohan et al., 1997; Staub and Serquen, 1996). Molecular markers are being used in conjunction with conventional methods in many plant breeding programs, including for MAS (Yi et al., 2004). Our use of MAS for EFB resistance resulted from difficulties in accurately phenotyping the disease response, the long incubation time for symptom development, and a desire to identify resistant seedlings without spreading the pathogen. In contrast to multiple genes, resistance conferred by a dominant allele at a single locus should be easier to combine with important horticultural traits. Presently, RAPD markers linked to 'Gasaway' and 'Ratoli' resistance are being used in MAS for EFB resistance in the hazelnut breeding program at OSU (Mehlenbacher et al., 2004; Sathuvalli et al., 2011). Eventually we envision marker-aided pyramiding of resistance genes. The addition of Georgian selection OSU 759.010 to our group of resistant accessions 


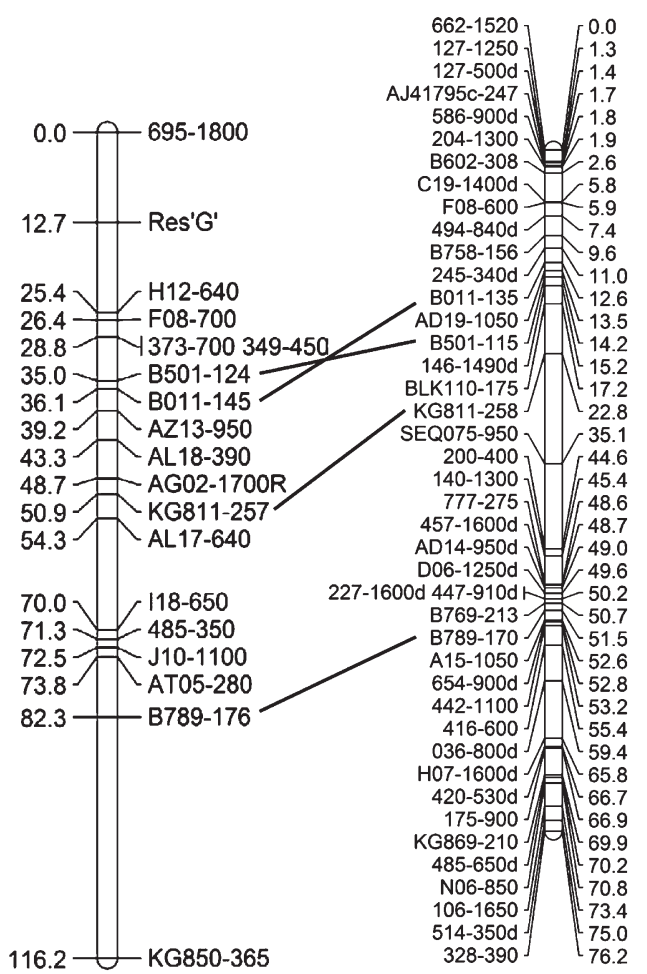

Fig. 3. Comparison of maps for linkage group 2 of Corylus avellana in OSU $759.010 \times$ OSU 653.068 (progeny 01032, left) and OSU 252.146 × OSU 414.062 (right). The Georgian resistance locus is indicated as Res ' $G$ '.

Table 3. Chi-square contributions of markers on linkage group 2 in Corylus avellana progeny 01032 segregating for eastern filbert blight resistance from Georgian selection OSU 759.010. ${ }^{z}$

\begin{tabular}{|c|c|c|}
\hline Locus $^{y}$ & Pairs (no.) & $\chi^{2}$ \\
\hline $695-1800$ & 15 & 0.688 \\
\hline Resistance & 14 & 0.711 \\
\hline H12-640 & 17 & 0.162 \\
\hline F08-700 & 17 & 0.195 \\
\hline $349-450$ & 17 & 0.168 \\
\hline $373-650$ & 17 & 0.168 \\
\hline B501-124 & 17 & 0.197 \\
\hline B011-145 & 17 & 0.219 \\
\hline AZ13-950 & 17 & 0.668 \\
\hline AL18-390 & 17 & 1.212 \\
\hline AG02-1700R & 17 & 2.826 \\
\hline KG811-257 & 17 & 1.625 \\
\hline AL17-640 & 17 & 0.511 \\
\hline I18-650 & 18 & 0.120 \\
\hline $485-350$ & 18 & 0.124 \\
\hline $\mathrm{J} 10-1100$ & 17 & 0.126 \\
\hline AT05-280 & 16 & 0.170 \\
\hline B789-176 & 16 & 0.161 \\
\hline KG850-365 & 5 & 0.029 \\
\hline \multicolumn{3}{|c|}{$\begin{array}{l}{ }^{\mathrm{z}} \text { Chi-square contributions were calculated by the software package } \\
\text { JoinMap } 4.0 \text { (Van Ooijen and Voorrips, 2006). } \\
\text { y Designations for random amplified polymorphic DNA markers are the } \\
\text { primer followed by the length of the polymorphic fragment in base } \\
\text { pairs. Primers with three digits are from the University of British } \\
\text { Columbia, Vancouver, BC, Canada. Primers with one or two letters are } \\
\text { from Eurofins MWG Operon, Huntsville, AL. Markers that begin with } \\
\text { the letter B or KG are simple sequence repeat markers. }\end{array}$} \\
\hline
\end{tabular}

expands the genetic base of our breeding population, and the identification of linked RAPD markers will facilitate the selection of resistant seedlings. Eventually this new source of resistance and linked markers will be used to pyramid different EFB resistance genes and to develop new cultivars with durable resistance.

Three resistant seedlings, three susceptible seedlings, and the parents of the cross OSU 759.010 $\times$ OSU 653.068 were used in the initial screening of primers to identify RAPD markers linked to resistance. The identified potential markers were then screened on a large number of phenotypically well-characterized samples to confirm linkage. This strategy identifies more markers and overcomes commonly encountered problems associated with the bulked segregant analysis (BSA) approach (Michelmore et al., 1991). With BSA, the inclusion of recombinants or incorrectly phenotyped seedling in the bulks may prevent identification of linked markers (Chen et al., 2005). The major disadvantage associated with the present method is the chance identification of unlinked markers. RAPD fragments are amplified from different places in the genome (Williams et al., 1990), so it is not surprising that some of the initially identified markers turned out to be unlinked.

The present study identified 13 markers linked to resistance, and separate linkage maps were constructed for progenies 01032 and 01033 . The order and distances of markers in the two maps were in general agreement. Slight differences were expected because the maps were constructed based on recombination frequencies on homologous chromosomes in two populations (Grattapaglia and Sederoff, 1994). The markers 695-1800, 373-700, 349-450, H12-640, and F08-700 were easy to score and have potential for use in MAS. The marker H12640 may be less useful than the others, because it was monomorphic in one of our populations. Marker 695-1800 is robust and located on the distal side of the resistance locus, whereas three other markers $(373-700,349-450$, and F08-650) are proximal. Of the latter, 373-700 is robust, easier to score, and appears most suitable for MAS. The practical application of large-scale MAS in applied plant breeding programs requires high-throughput techniques and cost-effective markers that are consistent and easy to score. RAPD markers 695-1800 and 373700 meet these criteria.

Segregation distortion is a common phenomenon that affects diverse types of plants including annuals and perennials, and it is likely the result of many mechanisms (Lunde et al., 2006). Aberrant segregation ratios may arise from a variety of physiological or genetic causes and may result from differential transmission in either the male (Liedl and Anderson, 1993; Mangelsdorf and Jones, 1926) or female germline (Yanagihara et al., 1995) or post-zygotic selection before phenotypic evaluation (Gadish and Zamir 1986; Xu et al., 1997). In this study, we observed a peculiar deviation from Mendelian segregation in one of the two progenies. In progeny 01032, the disease response segregated three resistant:one susceptible and segregation at adjacent markers also deviated from the expected 1:1 ratio. However, in progeny 01033 , the disease response segregated at the expected ratio of one resistant:one susceptible, but there was a surplus of seedlings with bands present at closely linked markers. Resistant selection OSU 759.010 was the female parent of both progenies.

Lunde et al. (2006) compared segregation for resistance from 'Gasaway' and 'Zimmerman'. The RAPD markers linked to 'Gasaway' resistance can also be used to follow 'Zimmerman' resistance, and SSR fingerprints indicate that 'Zimmerman' is 
likely a hybrid between 'Barcelona' and 'Gasaway' (Gökirmak et al., 2009). However, Lunde et al. (2006) found that in crosses with susceptible selections, 'Gasaway' transmits resistance to half of its offspring, whereas 'Zimmerman' transmits resistance to $75 \%$ of its offspring. The reason for the two different ratios, 1:1 and 3:1, remains unknown. The cytological phenomenon associated with this differential segregation has not yet been studied but might provide a better understanding of the segregation for disease resistance from Georgian selection OSU 759.010.

Cytological aberrations, including reciprocal translocations and inversions, are also associated with distorted segregation. In a few common $C$. avellana cultivars, heterozygous translocations associated with reduced pollen fertility have been reported (Salesses, 1973; Salesses and Bonnet, 1988). This might provide an explanation for the distorted segregation associated with OSU 759.010 because this selection has reduced pollen fertility and is functionally male-sterile. Most of its catkins fall to the orchard floor in the fall, and the others fail to elongate. The effect of a heterozygous translocation on the post-zygotic development of female gametophytes is not clearly understood, and further studies will indeed be helpful to understand segregation distortion. Tonguç et al. (2003) showed extreme segregation distortion of RAPD markers associated with black rot resistance in Brassica oleracea L. derived from Brassica carinata A. Braun. Several resistant seedlings, selected from these two progenies, represent improvements over their Georgian parent. They have been used as parents in breeding but unfortunately are also functionally male-sterile. It is hoped that study of segregation for disease response and linked markers in these progenies will clarify the situation. Furthermore, observing the meiotic behavior of the OSU 759.010 and selected resistant offspring might elucidate the underlying cytological phenomena associated with segregation distortion. In particular, it might provide evidence of reciprocal translocations or inversions. We are presently unable to provide a plausible explanation for the difference in segregation ratios observed in the two progenies derived from a single maternal parent.

Errors in disease phenotyping, marker genotyping, and missing values potentially affect the construction of a linkage map (Hackett and Broadfoot, 2003). The ideal set of molecular marker data for construction of a linkage map should have no missing values, no genotyping errors, and expected ratios of marker segregation. Nonetheless, Hackett and Broadfoot (2003) showed that segregation distortion has little effect on the construction of a linkage map. Our data confirm this, because maps for the two progenies were very similar although marker segregation ratios for the progenies differed.

The pyramiding of EFB resistance from different sources will be aided by molecular markers linked to each resistance. The goal of R-gene pyramids is long-term durable resistance. Three EFB resistance loci have been mapped: 'Gasaway' to LG6, 'Ratoli' to LG7, and OSU 759.010 to LG2. The pyramiding of two or even three of these resistances should be straightforward by means of MAS using linked RAPD markers.

A comparison of LG2 genetic maps derived from progeny 01032 (OSU 759.010 resistance) and the population OSU $252.152 \times$ OSU 414.062 (Fig. 3) (Mehlenbacher et al., 2006) shows a flip in the order of SSR markers B501 and B011. Such slight differences are not unusual and are commonly attributed to errors in marker genotyping and missing values. The SSRs mapped in the progenies of OSU 759.010 are not close to the resistance locus. The development of additional SSR loci will add to the markers available for MAS. Other SSR markers on LG2 (AJ41795c, B602, and B758) were not polymorphic in our populations.

EFB is generally more severe in a field nursery in New Jersey than in Oregon. In New Jersey, 'Gasaway' trees showed a few small cankers on small twigs (Molnar et al., 2010b). The cankers have stromata, and the cankers expand slowly. Nearby 'Zimmerman' trees showed a few sunken cankers but no stromata, whereas OSU 759.010 became infected (cankers with stromata) but showed less disease than 'Tonda di Giffoni', which has a high level of quantitative resistance (T.J. Molnar, personal communication).

To conclude, OSU 759.010 transmits its resistance and RAPD markers linked to the resistance to $50 \%$ to $75 \%$ of its progeny. The resistance locus is on LG2, which is different from other identified resistances. Thus, OSU 759.010 has potential for use in EFB resistance breeding either singly or in combination with other sources of resistance in a pyramiding strategy.

\section{Literature Cited}

Bassil, N.V., R. Botta, and S.A. Mehlenbacher. 2005a. Microsatellite markers in hazelnut: Isolation, characterization and cross-species amplification. J. Amer. Soc. Hort. Sci. 130:543-549.

Bassil, N.V., R. Botta, and S.A. Mehlenbacher. 2005b. Additional microsatellite markers of the european hazelnut. Acta Hort. 686: 105-110.

Boccacci, P., A. Akkak, N.V. Bassil, S.A. Mehlenbacher, and R. Botta. 2005. Characterization and evaluation of microsatellite loci in european hazelnut (Corylus avellana L.) and their transferability to other Corylus species. Mol. Ecol. Notes 5:934-937.

Cameron, H.R. 1976. Eastern filbert blight established in the Pacific Northwest. Plant Dis. Rptr. 60:737-740.

Chen, H., S.A. Mehlenbacher, and D.C. Smith. 2005. AFLP markers linked to eastern filbert blight resistance from OSU 408.040 hazelnut. J. Amer. Soc. Hort. Sci. 130:412-417.

Chen, H., S.A. Mehlenbacher, and D.C. Smith. 2007. Hazelnut accessions provide new sources of resistance to eastern filbert blight. HortScience 42:466-469.

Coyne, C.J., S.A. Mehlenbacher, R.O. Hampton, J.N. Pinkerton, and K.B. Johnson. 1996. Use of ELISA to rapidly screen hazelnut for resistance to eastern filbert blight. Plant Dis. 80:1327-1330.

Coyne, C.J., S.A. Mehlenbacher, and D.C. Smith. 1998. Sources of resistance to eastern filbert blight in hazelnut. J. Amer. Soc. Hort. Sci. 123:253-257.

Davison, A.R. and R.M. Davidson, Jr. 1973. Apioporthe and Monochaetia cankers reported in western Washington. Plant Dis. Rptr. 57:522-523.

Devos, K.M. and M.D. Gale. 1992. The use of random amplified polymorphic DNA markers in wheat. Theor. Appl. Genet. 84:567572.

Food and Agriculture Organization of the United Nations. 2009. FAO production yearbook. 1 July 2011. <http://faostat.fao.org/>.

Gadish, I. and D. Zamir. 1986. Differential zygotic abortion in an interspecific Lycopersicon cross. Genome 29:156-159.

Gökirmak, T., S.A. Mehlenbacher, and N.V. Bassil. 2009. Characterization of european hazelnut (Corylus avellana) cultivars using SSR markers. Genet. Resources Crop Evol. 56:147-172.

Grattapaglia, D. and R. Sederoff. 1994. Genetic linkage maps of Eucalyptus grandis and Eucalyptus urophylla using a pseudo-testcross: Mapping strategy and RAPD markers. Genetics 137:1121-1137.

Gürcan, K. and S.A. Mehlenbacher. 2010. Development of microsatellite marker loci for european hazelnut (Corylus avellana L.) from ISSR fragments. Mol. Breed. 26:551-559. 
Gürcan, K., S.A. Mehlenbacher, N.V. Bassil, P. Boccacci, A. Akkak, and R. Botta. 2010. Development, characterization, segregation, and mapping of microsatellite markers for european hazelnut (Corylus avellana L.) from enriched genomic libraries and usefulness in genetic diversity studies. Tree Genet. Genomes 6:513-531.

Hackett, C.A. and L.B. Broadfoot. 2003. Effects of genotyping errors, missing values and segregation distortion in molecular marker data on the construction of linkage maps. Heredity 90:33-38.

Johnson, K.B., S.A. Mehlenbacher, J.K. Stone, and J.W. Pscheidt. 1996. Eastern filbert blight of european hazelnut-It's becoming a manageable disease. Plant Dis. 80:1308-1316.

Lasareishvili, L.N. 2003. Recommendation: Progressive technology for the development and care of intensive type hazelnut orchards. Georgian Scientific-Research Institute of Horticulture, Viticulture and Wine making of Georgian Academy of Agricultural Sciences, Tbilisi, Georgia.

Liedl, B. and N.O. Anderson. 1993. Reproductive barriers: Identification, uses and circumvention. Plant Breed. Rev. 11:11-154.

Lunde, C.F., S.A. Mehlenbacher, and D.C. Smith. 2000. Survey of hazelnut cultivars for response to eastern filbert blight inoculation. HortScience 35:729-731.

Lunde, C.F., S.A. Mehlenbacher, and D.C. Smith. 2006. Segregation for resistance to eastern filbert blight in progeny of 'Zimmerman' hazelnut. J. Amer. Soc. Hort. Sci. 131:731-737.

Mangelsdorf, P.C. and D.F. Jones. 1926. The expression of Mendelian factors in the gametophyte of maize. Genetics 11:423-455.

Mehlenbacher, S.A. 1994. Genetic improvement of hazelnut. Acta Hort. 351:23-28.

Mehlenbacher, S.A., R.N. Brown, J.W. Davis, H. Chen, N. Bassil, and D.C. Smith. 2004. RAPD markers linked to eastern filbert blight resistance in Corylus avellana. Theor. Appl. Genet. 108:651-656.

Mehlenbacher, S.A., R.N. Brown, E.R. Nouhra, T. Gökirmak, N.V. Bassil, and T.L. Kubisiak. 2006. A genetic linkage map for hazelnut (Corylus avellana L.) based on RAPD and SSR markers. Genome 49:122-133.

Mehlenbacher, S.A., M.M. Thompson, and H.R. Cameron. 1991. Occurrence and inheritance of resistance to eastern filbert blight in 'Gasaway' hazelnut. HortScience 26:410-411.

Meunier, J.R. and P.A.D. Grimont. 1993. Factors affecting reproducibility of random amplified polymorphic DNA fingerprinting. Res. Microbiol. 144:373-379.

Michelmore, R.W., I. Paran, and R.V. Kesseli. 1991. Identification of markers linked to disease resistance genes by bulked segregant analysis: A rapid method to detect markers in specific genomic regions using segregating populations. Proc. Natl. Acad. Sci. USA 88:9828-9832.

Mohan, M., S. Nair, A. Bhaqwat, T.G. Krishna, M. Yano, C.R. Bhatia, and T. Sasaki. 1997. Genome mapping, molecular markers and marker-assisted selection in crop plants. Mol. Breed. 3:87103.

Molnar, T.J., S.N. Baxer, and J.C. Goffreda. 2005. Accelerated screening of hazelnut seedlings for resistance to eastern filbert blight. HortScience 40:1667-1669.

Molnar, T.J., J.C. Goffreda, and C.R. Funk. 2010a. Survey of Corylus resistance to Anisogramma anomala from different geographic locations. HortScience 45:832-836.

Molnar, T.J., J. Capik, S. Zhao, and N. Zhang. 2010b. First report of eastern filbert blight on Corylus avellana 'Gasaway' and 'VR20-11' caused by Anisogramma anomala in New Jersey. Plant Dis. 94:1265. Pinkerton, J.N., K.B. Johnson, D.E. Aylor, and J.K. Stone. 2001. Spatial and temporal increase of eastern filbert blight in european hazelnut orchards in the Pacific Northwest. Phytopathology 91:1214-1223.

Pinkerton, J.N., K.B. Johnson, S.A. Mehlenbacher, and J.W. Pscheidt. 1993. Susceptibility of european hazelnut clones to eastern filbert blight. Plant Dis. 77:261-266.

Pinkerton, J.N., K.B. Johnson, J.K. Stone, and K.L. Ivors. 1998a. Factors affecting the release of ascospores of Anisogramma anomala. Phytopathology 88:122-128.

Pinkerton, J.N., K.B. Johnson, J.K. Stone, and K.L. Ivors. 1998b. Maturation and seasonal discharge pattern of ascospores of Anisogramma anomala. Phytopathology 88:1165-1173.

Pinkerton, J.N., K.B. Johnson, K.M. Theiling, and J.A. Griesbach. 1992. Distribution and characteristics of the eastern filbert blight epidemic in western Oregon. Plant Dis. 76:1179-1182.

Pinkerton, J.N., J.K. Stone, S.J. Nelson, and K.B. Johnson. 1995. Infection of european hazelnut by Anisogrammaanomala: Ascospore adhesion, mode of penetration of immature shoots, and host response. Phytopathology 88:1260-1268.

Pscheidt, J.W. 2006. Potential EFB control programs. Proc. Nut Growers Soc. Oregon Washington British Columbia 91:72-78.

Salesses, G. 1973. Etude cytologique du genre Corylus. Mise en évidence d'une translocation hétérozygote chez quelques variétés de noisetier cultivé (Corylus avellana L.) à fertilité pollinique réduite. Annales de 1'Amélioration des Plantes 23:59-66.

Salesses, G. and A. Bonnet. 1988. Etude cytogénétique d'hybrides entre variétés de noisetier (Corylus avellana L.) porteuses d'une translocation à l'état hétérozygote. Cytologia (Tokyo) 53:407-413.

Sathuvalli, V.R., H. Chen, S.A. Mehlenbacher, and D.C. Smith. 2011. DNA markers linked to eastern filbert blight resistance in 'Ratoli' hazelnut (Corylus avellana L.). Tree Genet. Genomes 7:337-345.

Sathuvalli, V.R., S.A. Mehlenbacher, and D.C. Smith. 2010. Response of hazelnut accessions to greenhouse inoculation with Anisogramma anomala. HortScience 45:1116-1119.

Staub, J. and F. Serquen. 1996. Genetic markers, map construction, and their application in plant breeding. HortScience 31:729-741.

Stone, J.K., K.B. Johnson, J.N. Pinkerton, and J.W. Pscheidt. 1992. Natural infection period and susceptibility of vegetative seedlings of european hazelnut to Anisogramma anomala. Plant Dis. 76:348-352.

Tonguç, M., E.D. Earle, and P.D. Griffiths. 2003. Segregation distortion of Brassica carinata derived black rot resistance in Brassica oleracea. Euphytica 134:269-276.

Van Ooijen, J.W. and R.E. Voorrips. 2006. JoinMap 4.0, Software for the calculation of genetic linkage maps. Kyazama, Wageningen, The Netherlands.

Welsh, J. and M. McClelland. 1990. Fingerprinting genomes using PCR with arbitrary primers. Nucleic Acids Res. 18:7213-7218.

Williams, J., A. Kubelik, K. Livak, J. Rafalski, and S. Tingey. 1990. DNA polymorphisms amplified by arbitrary primers are useful as genetic markers. Nucleic Acids Res. 18:6531-6535.

Xu, Y., L. Zhu, J. Xiao, N. Huang, and S.R. McCouch. 1997. Chromosomal regions associated with segregation distortion of molecular markers in $\mathrm{F}_{2}$, backcross, doubled haploid, and recombinant inbred populations in rice (Oryza sativa L.). Mol. Gen. Genet. 253:535-545. Yanagihara, S., S.R. McCouch, K. Ishikawa, Y. Ogi, K. Maruyama, and H. Ikehashi. 1995. Molecular analysis of the inheritance of S-5 locus, conferring wide compatibility in Indica/Japonica hybrids of rice (O. sativa L.). Theor. Appl. Genet. 90:182-188.

Yi, G., S.K. Lee, Y.K. Hong, T.Y.C. Cho, M.H. Nam, S.C. Kim, S.S. Han, G.L. Wang, T.R. Hahn, P.C. Ronald, and J.S. Jeon. 2004. Use of Pi5 ( $\mathrm{t}$ ) markers in marker-assisted selection to screen for cultivars with resistance to Magnaporthe grisea. Theor. Appl. Genet. 109: 978-985. 